\title{
Stress evolution in elastic-plastic electrodes during electrochemical processes: A numerical method and its applications
}

\author{
Jici Wen ${ }^{\mathrm{a}, \mathrm{c}}$, Yujie Wei ${ }^{\mathrm{a}, \mathrm{c}, *}$, Yang-Tse Cheng ${ }^{\mathrm{b}}$ \\ a State Key Laboratory of Nonlinear Mechanics (LNM), Institute of Mechanics, Chinese Academy of Sciences, Beijing, 100190, China \\ ${ }^{\mathrm{b}}$ Department of Chemical and Materials Engineering, University of Kentucky, Lexington, KY 40506, USA \\ ' School of Engineering Sciences, University of Chinese Academy of Sciences, Beijing, 100049, China
}

\section{A R T I C L E I N F O}

\section{Article history:}

Received 28 December 2017

Revised 13 April 2018

Accepted 13 April 2018

Available online 21 April 2018

\section{Keywords:}

Battery mechanics

Electrochemical-mechanical coupling

Interfacial delamination

In situ stress measurement

Finite element method

\begin{abstract}
A B S T R A C T
Monitoring in real time the stress state in high capacity electrodes during charge-discharge processes is pivotal to the performance assessment and structural optimization of advanced batteries. The wafer curvature measurement technique broadly employed in thinfilm industry, together with stress analysis using the Stoney equation, has been successfully adopted to measure in situ the stress in thin film electrodes. How large plastic deformation or interfacial delamination during electrochemical cycles in such electrodes affects the applicability of Stoney equation remains unclear. Here we develop a robust electrochemical-mechanical coupled numerical procedure to investigate the influence of large plastic deformation and interfacial failure on the measured stress in thin film electrodes. We identify how the constitutive behavior of electrode materials and film-substrate interfacial properties affect the measured stress-capacity curves of electrodes, and hence establish the relationship of electrode material parameters with the characteristics of stress-capacity curves. Using Li-ions batteries as examples, we show that plastic deformation and interfacial delamination account for the asymmetric stress-capacity loops seen in in situ stress measurements. The methods used here, along with the finite-element code in the supplementary material, may be used to model the electrode behavior as a function of the state of charge.
\end{abstract}

(c) 2018 Elsevier Ltd. All rights reserved.

\section{Introduction}

High charge capacity anode materials like Si and Sn have attracted great interests in recent years (Bourderau et al., 1999; Liang et al., 2014; Huggins, 1999; Winter and Besenhard, 1999). One major tradeoff arisen from the high capacity is the resulted high stress. Lithiation and de-lithiation in anode materials inevitably result in huge volume expansion and contraction, lead to high internal stresses and, thus, poor cycle life of high capacity anode batteries (Shi et al., 2016; Klinsmann et al., 2016; Zhang et al., 2016). Many strategies were proposed to prolong the service duration of anode materials. One popular approach was to optimize the microstructure of the materials (Szczech and Jin, 2011; Zhang et al., 2017) to alleviate internal stress induced by volume expansion. To examine the effectiveness of such structural optimization, we desire to have

\footnotetext{
* Corresponding author at: State Key Laboratory of Nonlinear Mechanics (LNM), Institute of Mechanics, Chinese Academy of Sciences, Beijing, 100190, China.

E-mail address: yujie_wei@Inm.imech.ac.cn (Y. Wei).
} 

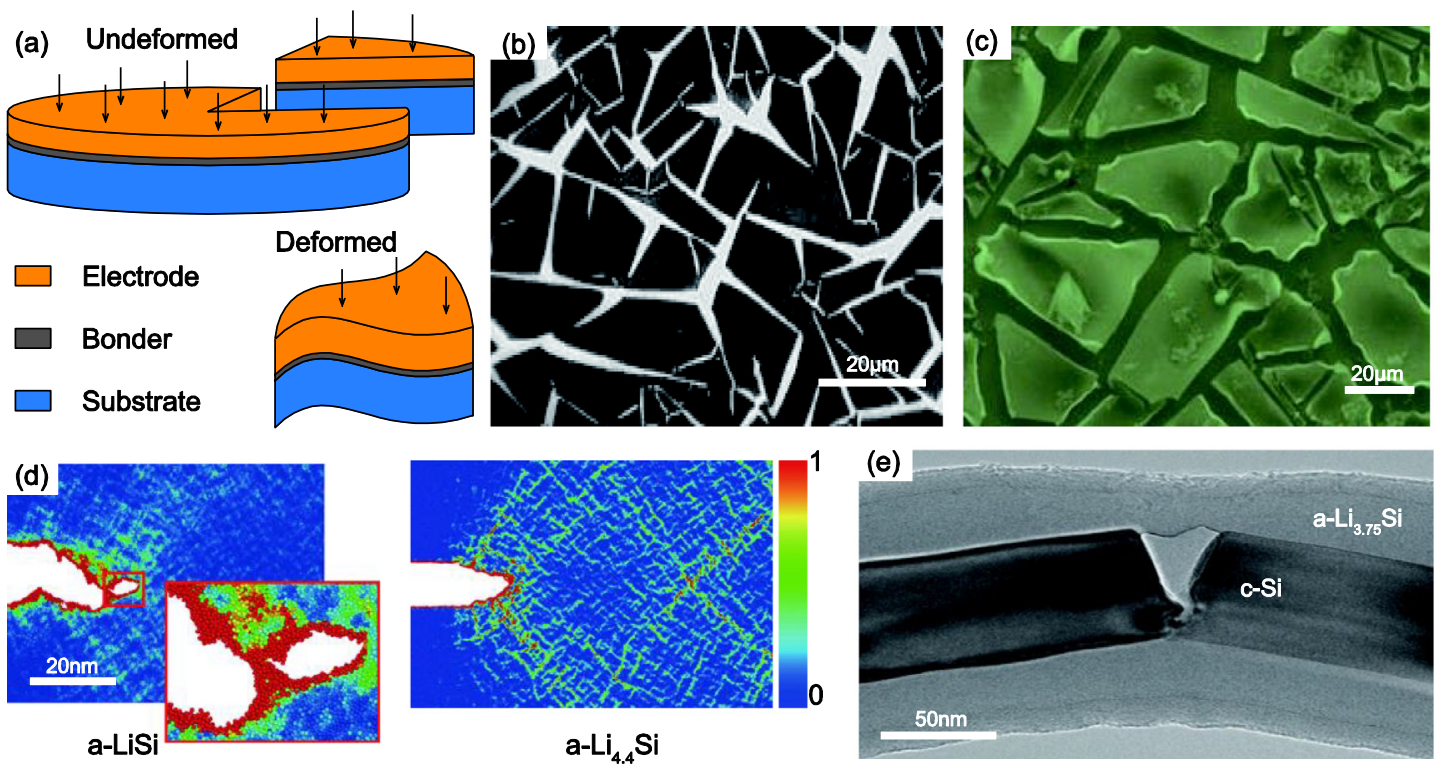

Fig. 1. Illustrations of high capacity battery electrodes undergone large deformation and interfacial failure: (a) A film-bonder-substrate system for in situ curvature monitoring. (b) Optical micrograph showing micro-cracks in amorphous silicon thin film electrode after charge and discharge. (c) SEM picture to show interfacial delamination and cracking in the thin film electrode after a certain amount of cycles (b and c, Courtesy of Yang et al., 2018). (d) Molecular dynamic simulations showed brittle to ductile transition, with cavitation dominated failure in low lithium concentration amorphous silicon (left) versus micro-shearing in high lithium concentration amorphous silicon (right, Courtesy of Ding et al., 2015). (e) Experimental observation on ductile deformation in amorphous silicon with high lithium concentration against brittle fracture in crystalline silicon (Courtesy of Wang et al., 2015).

an accurate and real time measurement of stress in high capacity electrodes (Sethuraman et al., 2010, 2012; Duan and Wei, 2017). Real time stress is also indispensable to better our understanding about the electrochemical-mechanical processes and is helpful for faithful model development for electrode materials.

Stresses in thin film electrodes may be measured by monitoring the bending of the electrode-substrate bilayer and using the Stoney equation. A typical battery setup for such in situ stress measurement is shown in Fig. 1a, which consists of a thin film, a bonder layer, and a substrate. Using this method, Sethuraman et al. (2010, 2012) showed how stress changes in Si- and graphite-based anode film materials during charging and discharging. Later on, Soni et al. (2011) and Haftbaradaran et al. (2012) used the same method to investigate the stress-capacity behavior of patterned electrode islands, aimed to illustrate the influence of interfacial failure between electrode islands and the substrate on capacity degradation (He et al., 2012). Recently, Pharr et al. (2016) measured stresses in sputter-deposited amorphous Ge thin films as the electrode material. The stress-capacity curves from different research groups all showed a hysteresis loop, which was caused by irreversible deformation during charge-discharge cycles. In addition, the electrode-substrate interface may be subjected to excessive deformation and may fail during cycling. According to the in situ tests, fracture and delamination of the film electrode from substrate, as shown in Fig. 1b, were broadly observed (e.g., Maranchi et al., 2006; Yang et al., 2018). Such interfacial deformation will inevitably influence the stress-capacity curve. Connecting the properties of the interface and the anode material with the measured curvature-capacity curve is hence crucial to determine the actual stress in the electrode material. More importantly, we may use the curvature-capacity curves to extract the interface and material properties.

A typical route to obtain material parameters in constitutive models is to compare the predicted stress-capacity response from the models with available experimental data. Following this strategy, different groups have developed constitutive models and the numerical methods for electrode materials to decipher the materials' properties from the stress-capacity response. For example, Bhandakkar et al. (2010) compared the stress-capacity curves of Sn films from numerical analysis and experimental data, and estimated the interfacial strength. Zhao et al. (2012) and Pharr et al. (2013) studied the mechanical properties of electrode materials using first-principle calculations, and compared their results with measured stress-capacity curves. Jia and Li (2016) and Wen et al. (2018) explored the influence of concentration-dependent elasticity on stresses in spherical particles and film-substrate structures, respectively. Zhao et al. (2011, 2012) and Brassart et al. (2013) reported how the electrode materials' plastic flow and shakedown were related to the characteristics in the stress-capacity curves. Bower et al. (2011) predicted the evolution of stress and potential for a one-dimension Li-ion half-cell with a Si anode by considering finite strain, plastic flow, and electric current flow. By taking an analogy between diffusion and heat conduction, Yang et al. (2014) presented a chemical-mechanical model to investigate the lithiation-induced phase transformation, morphological evolution, stress generation and fracture in crystalline silicon nanowires. Levitas and Attariani (2014) analyzed the anisotropic compositional expansion in elastic-plastic anode materials. The work by Anand (2012), Di Leo et al. 
(2014) and Chester et al. (2015) coupled Cahn-Hilliard-type diffusion with large elastic-plastic deformation for anode materials' diffusion-induced swelling for lithium-ion batteries and applied the model to phase-separating problems.

Since the reliability of a stress-capacity curve depends on the applicability of the Stoney equation for in situ measurement (Stoney, 1909), it is important to know how the deformation mechanisms in the materials and the interfaces affect those curves. It is known that large deformation and interfacial failure are not taken into account by the Stoney equation. Concentration-dependent material parameters will make theoretical modeling more difficult. Physically-based constitutive models, combined with robust numerical methods, may help surmount many of these challenges. In this paper, we first present a robust numerical procedure and its finite element implementation in Section 2. The elastic-plastic constitutive equations for diffusion-induced stresses are also shown in Section 2. In Section 3, we show how lithium concentrationdependent elastic-plastic properties affect the stress-capacity curves of thin film batteries. In Section 4, we demonstrate the influence of the interfacial delamination on the stress-capacity of thin film electrodes. We conclude in Section 5 with final remarks and discussions.

\section{The theoretical model}

If the anode material is crystalline, phase change may occur within the first few cycles till the anode material transforms to an amorphous state. That is commonly seen in Si anodes. Several groups have developed constitutive models to capture the associated electrochemical-mechanical processes (Bower et al., 2011; Anand, 2012; Di Leo et al., 2014; Chester et al., 2015), and the physical processes taken into account include (1) the diffusion mechanism governed by concentration and electric field, (2) concentration variation induced volume change, (3) concentration variation associated with elastic-plastic deformation, and (4) stress resulted from deformation. In this paper, we aim to add more physical mechanisms by accounting for (a) concentration dependent flow stress, (b) competition between plasticity in the electrodes and deformation at the interfaces between electrodes and substrates, and (c) effects of electrode plasticity and interfacial failure on stress measurement using the Stoney equation. We are motivated to taking these mechanisms into consideration by recent experimental observations, as seen in Fig. 1b-e. In particular, the results from Fig. 1d and e suggest the need for the concentrationdependent elastic-plastic behavior of electrode materials. An open source and robust finite-element procedure is provided for small elastic yet large plastic deformation occurring in most high capacity anode materials. The model is implemented in the commercial FE package ABAQUS (ABAQUS/Standard, 2013) as a user-element (See supplementary material). The numerical method can also be applied for nearly incompressible anode materials, which can be used for any complex structure, like nanowire (Chan et al., 2008) and nano-island (Soni et al., 2011; Duan and Wei, 2017).

\subsection{Governing equations}

In an anode material during changing (discharging), the governing equations, associated with electrochemical reaction and mechanical deformation, are presented here.

1. The local force balance equation: It is assumed that the chemical reaction rate is much slower than stress waves, and hence we have force equilibrium in the form of

$$
\frac{\partial \sigma_{i j}}{\partial x_{j}}=0
$$

where $\sigma_{i j}$ is the Cauchy stress at a material point $x_{j}$.

2. The diffusion equation: At any time $t$, the lithium concentration $\phi(x, t)$ at a material point $x_{i}$ in the electrode structure of volume $v$ is governed by the diffusion equation (Carslaw and Jaeger, 1959)

$$
\frac{\partial \phi}{\partial t}+\nabla \cdot \boldsymbol{J}=0
$$

The concentration and flux boundary conditions are respectively given as

$$
\phi=\tilde{\phi} \text { on } \Gamma_{1} \text { and } \boldsymbol{J} \cdot \boldsymbol{n}=J_{i} n_{i}=-J^{s} \text { on } \Gamma_{2},
$$

where the sum of $\Gamma_{1}$ and $\Gamma_{2}$ represents the overall surface of the volume $v$, and $\boldsymbol{n}$ is the surface normal of $\Gamma_{2}$, and $J^{s}$ is the surface flux. The galvanostatic current boundary condition $J^{s}$ is related to the current density $i^{s}$ via $J^{s}=\frac{i^{s}}{F}$ (Cheng and Verbrugge, 2009) for $F$ being the Faraday constant. The concentration $\phi$ is associated with compound phase $A_{\psi} B$ in the electrode where $A$ and $B$ indicate the lithium and the anode electrode material, respectively, and the subscript $\psi$ indicates the lithium fraction ranging from 0 to $\psi_{\max }$, and the maximum lithium fraction $\psi_{\max }$ is known as $\psi_{\max }=4.4$ $\left(L i_{4.4} S i\right.$, Boukamp et al., 1981). The connection between $\phi$ and $\psi$ is defined by

$$
\phi=\frac{\psi}{V_{\psi} N_{a}},
$$

where $V_{\psi}$ is the volume of the compound $A_{\psi} B$ and is a function of $\psi$, and $N_{a}$ is Avogadro's number. Taking the lithiumsilicon battery system as an example, we may write the volume of the compound $L i_{\psi} S i$ as (Fan et al., 2013; He et al., 
2012)

$$
V_{\psi}=\left(1+3.2 \frac{\psi}{\psi_{\max }}\right) \times 10^{-28} \mathrm{~m}^{3} .
$$

Considering $\psi$ as a continuous variable, we can rewrite the diffusion equation in terms of $\psi$ as

$$
\frac{V_{\psi}-\psi V_{\psi}^{\prime}}{V_{\psi}^{2} N a} \frac{\partial \psi}{\partial t}+\nabla \cdot \boldsymbol{J}=0(\text { in } v)
$$

3. The constitutive relationship for the Li-ions flux: The Li-ions flux $\boldsymbol{J}$ is commonly used as a function of the gradient of a stress-dependent chemical potential $\mu$ (Li, 1978; Yang, 2005; Zhang et al., 2008)

$$
\boldsymbol{J}=-\frac{D(\phi, \boldsymbol{x})}{R T} \nabla \mu\left(\phi, \sigma_{h}, \boldsymbol{x}, t\right)
$$

As the exact dependence of $\mu$ on $\sigma$ remains debatable, we adopt a simple form (Wei et al., 2010)

$$
\mu\left(\phi, \sigma_{h}, \boldsymbol{x}, t\right)=\mu_{0}+R T \ln \frac{\phi}{\phi_{\max }}-\Omega \sigma_{h},
$$

where $\mu_{0}$ is the referential potential, $\phi_{\max }$ is the maximum lithium concentration and $\Omega$ the lithium ions partial molar volume and $\sigma_{h}$ the hydrostatic stress, i.e., $\left(\sigma_{x x}+\sigma_{y y}+\sigma_{z z}\right) / 3$. The second term represents an entropic contribution to the free-energy, while the third term is the work done by the applied stress. Combining Eqs. (6) and (7), the flux equation can be reformulated as

$$
\boldsymbol{J}=-\frac{D(\phi, \boldsymbol{x})}{\phi} \nabla \phi(\boldsymbol{x}, t)+\frac{D(\phi, \boldsymbol{x}) \Omega}{R T} \nabla \sigma_{h} .
$$

We consider a linear dependence of the diffusivity on concentration and assume isotropic diffusion in amorphous electrode materials, $D(\phi, \boldsymbol{x})=D_{0} \phi$.

4. The constitutive relationship for the Cauchy stress: The rate-independent elastic-plastic constitutive relationship (coupled with diffusion) in the incremental form is then given as

$$
d \sigma_{i j}^{J}=C_{i j k l} d \varepsilon_{k l}^{e}
$$

where $d \sigma_{i j}^{J}$ is an objective increment of the Cauchy stress, and $C_{i j k l}$ is the fourth-order elastic modulus tensor, which depends on the lithium concentration $\psi$. Note that the elastic strain is small for most high capacity anode material and the amorphous anode material is taken to be isotropic, then $C_{i j k l}$ only depend on Young's modulus $E(\psi)$ and the Poisson's ratio $v$. The Young's modulus $E(\psi)$ also varies slightly during the lithiation process (Shenoy et al., 2010; Sethuraman et al., 2010). The usual stretching tensor increment $d \varepsilon_{k l}^{m}$ can be composed of an elastic deformation part $d \varepsilon_{k l}^{e}$ and a plastic deformation part $d \varepsilon_{k l}^{p l}$, and $d \varepsilon_{k l}^{m}=d \varepsilon_{k l}^{e}+d \varepsilon_{k l}^{p l}$. For small elastic yet large plastic deformation, we used the loading/unloading criterion by accounting for the usual complementarity condition

$$
\Phi \leq 0, \lambda \geq 0, \lambda \Phi=0,
$$

where $\lambda$ is a plastic multiplier and $\Phi\left(\sigma_{i j}, \bar{\varepsilon}^{p}\right)$ is the yield function which depends on both the current stress and the equivalent plastic strain $\bar{\varepsilon}^{p}, \bar{\varepsilon}^{p}=\int \sqrt{\frac{2}{3} d \varepsilon_{k l}^{p l} \cdot d \varepsilon_{k l}^{p l}}$. We write it in the form of

$$
\Phi\left(\sigma_{i j}, \bar{\varepsilon}^{p}\right)=\sqrt{\frac{3}{2}\left(\sigma_{i j}-\frac{1}{3} \sigma_{i j} \delta_{i j}\right)\left(\sigma_{\mathrm{ij}}-\frac{1}{3} \sigma_{i j} \delta_{i j}\right)}-\sigma_{y}\left(\bar{\varepsilon}^{p}, \psi\right) .
$$

The second term at the right hand side of Eq. (11) indicates that the yield strength is a function of both $\bar{\varepsilon}^{p}$ and the lithium fraction $\psi$. A bilinear hardening modulus is used here,

$$
\sigma_{y}\left(\bar{\varepsilon}^{p}, \psi\right)=\sigma_{y 0}(\psi)+\frac{E(\psi) E_{t}(\psi)}{E(\psi)-E_{t}(\psi)} \bar{\varepsilon}^{p},
$$

where $\sigma_{y 0}(\psi)$ is the initial yield stress and $E_{t}(\psi)$ is the hardening modulus. Using the $J_{2}$ flow rule, we have

$$
d \varepsilon_{i j}^{p l}=\lambda \frac{\partial \Phi}{\partial \sigma_{i j}}
$$

Eq. (13) is used to determine the plastic strain increment.

5. Objective variables for finite deformation: The objective stress rate is defined by the Jaumann stress rate (McMeeking and Rice, 1975) as

$$
\dot{\sigma}_{i j}^{J}=\dot{\sigma}_{i j}+\sigma_{i k} W_{k j}-W_{i k} \sigma_{k j},
$$


where $\dot{\sigma}_{i j}$ is the time rate of Cauchy stress, $W_{i j}=\left(L_{i j}-L_{j i}\right) / 2$ is the spin tensor, $L_{i j}=\frac{\partial v_{i}}{\partial x_{j}}=\dot{\boldsymbol{F}}^{-1}$ is the velocity gradient for $F_{i j}=\frac{\partial x_{i}}{\partial X_{j}}$ being the deformation gradient, and $\dot{\sigma}_{i j}^{J}$ is the Jaumann stress rate, which can then be used to objectively describe the stress and strain rate $\left(\dot{\varepsilon}_{k l}\right)$ relation. By considering large plastic strain, we take the total logarithmic strain measure $\left[\boldsymbol{\varepsilon}_{n+1}\right]$ at time $n+1$ during the standard interval $\left[t_{n}, t_{n+1}\right]$ by

$$
\left[\boldsymbol{\varepsilon}_{n+1}\right]=\left[\boldsymbol{\varepsilon}_{n}\right]+[\boldsymbol{\Delta} \overline{\boldsymbol{\varepsilon}}]
$$

In Eq. (15), $[\boldsymbol{\Delta} \bar{\varepsilon}]$ is a rotation-neutralized strain increment from time $t_{\mathrm{n}}$ to $t_{\mathrm{n}+1}$. Unless stated otherwise, a parameter with a top bar is defined in the rotated coordinate. Hughes (1984) used the approximate 2nd-order calculation for $[\boldsymbol{\Delta} \overline{\boldsymbol{\varepsilon}}]$, which is given as

$$
[\boldsymbol{\Delta} \overline{\boldsymbol{\varepsilon}}]=\left[\boldsymbol{R}_{n+1 / 2}\right]^{T}[\Delta \boldsymbol{D}]\left[\boldsymbol{R}_{n+1 / 2}\right]
$$

where $\Delta \boldsymbol{D}=\operatorname{sym}\left(\frac{\partial \Delta \boldsymbol{u}}{\partial \boldsymbol{x}_{n+1 / 2}}\right)$ is the rotation-neutralized stretch increment tensor relative to the neutralized positions $\boldsymbol{x}_{n+1 / 2}=\frac{1}{2}\left(\boldsymbol{x}_{n}+\boldsymbol{x}_{\mathrm{n}+1}\right)$, and $\left[\boldsymbol{R}_{n+1 / 2}\right]$ comes from the decomposition of the deformation gradient $F_{i j}$ in the following,

$$
F_{i j}=R_{i k} U_{k j} \text {, and } R_{j i} R_{i j}=\delta_{i j}
$$

Here $\boldsymbol{R}$ is a rotational tensor and $\boldsymbol{U}$ is the right stretch tensor. The updated objective stress defined in the rotated coordinate is given as $\left[\overline{\boldsymbol{\sigma}}_{n+1}\right]=\left[\overline{\boldsymbol{\sigma}}_{n}\right]+[\Delta \overline{\boldsymbol{\sigma}}]$. This is a simple yet powerful algorithm for large inelastic deformation. The explicit expression of $R_{i j}$ in terms of quaternion parameters is also available (Hughes and Simo, 1998; Crisfield, 1997).

\subsection{Numerical procedures}

Now we layout the variational forms of the two partial differential Eqs. (1) and (5) for finite-element implementation.

1 The principle of virtual work $\delta W_{m}$ for mechanical deformation and its differentiation $d \delta W_{m}$ are given as

$$
\delta W_{m}=\int_{v} \sigma_{i j} \delta D_{i j} d v-\int_{v} f_{i}^{B} \delta u_{i} d v=0
$$

and

$$
d \delta W_{m}=\int_{v} \delta D_{i j} \frac{\partial \sigma_{i j}}{\partial \varepsilon_{k l}{ }^{m}} d \varepsilon_{k l}^{m} d v+\int_{v} \sigma_{i j}\left(\frac{\partial \delta u_{k}}{\partial x_{i}} \frac{\partial d u_{k}}{\partial x_{j}}-2 \delta D_{i k} d D_{k j}\right) d v+\int_{v}\left\{\delta D_{i j} \frac{\partial \sigma_{i j}}{\partial \psi} d \psi\right\} d v
$$

respectively.

2 The variational form $\delta W_{d}$ of the diffusion equation (Eq. (5)) and its differentiation is given, in turn, as

$$
\delta W_{d}=\int_{V} \delta \psi \frac{V_{\psi}-\psi V_{\psi}{ }^{\prime}}{V_{\psi}{ }^{2} N_{a}} \frac{\partial \psi}{\partial t} d v-\int_{V} \delta \psi_{, i} J_{i} d v-\int_{S} J^{s} \delta \psi d s=0
$$

and

$$
\begin{aligned}
d \delta W_{d}= & \int_{v} \delta \psi H(\psi) \frac{\partial d \psi}{\partial t} d v+\int_{v} \delta \psi_{, i} D_{0} H(\psi) d \psi_{, i} d v-\int_{v} \delta \psi_{, i} \frac{D_{0} \Omega}{R T} \sigma_{h, i} H(\psi) d \psi d v \\
& -\int_{v}\left[\delta \psi_{, i} G_{, m} d u_{m, i}+\delta \psi_{, m} G_{, i} d u_{m, i}\right] d v
\end{aligned}
$$

respectively. Here $H(\psi)=\frac{\partial \phi}{\partial \psi}=\frac{V_{\psi}-\psi V_{\psi}{ }^{\prime}}{V_{\psi}{ }^{2} N_{a}}$ and $G_{, i}=D_{0} H(\psi) \psi_{, i}-\frac{D_{0} \Omega}{R T} \frac{\psi}{V_{\psi} N_{a}} \sigma_{h, i}$. The first three terms on the RHS of Eq. (21) are associated with diffusion, and the last one is resulted from finite deformation.

\section{Plastic response in the electrode materials}

The film-bonder-substrate system (see Fig. 1a) is a typical structure used for in situ stress measurements during the charge/discharge process. The electrode film adhered to the substrate, when expands or contracts during the charge/discharge process, governs the substrate's curvature evolution. The film's biaxial stress $\sigma_{m}$ can be deduced from the substrate's curvature $\kappa$ using the Stoney equation (Stoney, 1909)

$$
\sigma_{m}=\frac{E_{s} h_{s}^{2}}{6\left(1-v_{s}\right) h_{f}} \kappa
$$

where $E_{S}$ and $v_{s}$ are the substrate's elastic modulus and Poisson's ratio, respectively, and the thickness of the substrate and the film are $h_{s}$ and $h_{f}$ respectively. The validity of the above equation is contingent on the following assumptions: (a) $h_{f} \ll h_{s}$, (b) $h_{f}$ remains nearly unchanged during the deformation, and (c) the interface between the film and the substrate is non-deformable and of zero-thickness. Previously, we demonstrated that even for elastic deformation in the electrode film, 
comparable $h_{f}$ and $h_{s}$ will give rise to significant differences between the true stress in the electrode film and that predicted based on Eq. (22) (Wen et al., 2018). To address the influence of assumptions (b) and (c) on real stress assessment, it is desired to solve the electrochemical-mechanical coupled problems within the finite deformation scheme, consider the plastic response of the elastic-plastic film electrode, and account for possible interfacial deformation.

In mimicking a typical in situ stress measurement, we consider an amorphous silicon film electrode constrained by the substrate with a thickness-radius ratio $\frac{h_{s}}{R_{s}}=0.2$. The thickness of the substrate is $h_{s}=60 \mu \mathrm{m}$ in the simulation. The electrode film's thickness $h_{f}$ is rather small, $\frac{h_{f}}{h_{s}}=0.002$. Its elastic modulus varies linearly with the Li-concentration (Shenoy et al., 2010)

$$
E_{f}=E_{0}+\frac{\psi(t)}{1+\psi(t)} m E_{0}
$$

where $E_{0}$ is the modulus of pure silicon and $m$ is a coefficient. The initial elastic modulus, the slope and the Poisson's radio are $E_{0}=90.13 \mathrm{GPa}, m=-0.79$ and $v_{f}=0.22$, in turn. The diffusivity is taken as $D_{0}=1.0 \times 10^{-16} \mathrm{~m}^{2} / \mathrm{s}($ Pan et al., 2015), and the lithium ions partial molar volume is $\Omega=3.497 \times 10^{-6} \mathrm{~m}^{3} / \mathrm{mol}$ (Zhang et al., 2007). For the substrate, its elastic modulus and Poisson's radio are $E_{s}=162 \mathrm{GPa}$ and, $v_{s}=0.26$ (Brantley, 1973), respectively. We consider a charging condition that a constant current density of $5 \mu \mathrm{A} / \mathrm{cm}^{2}$ (ca. C/24 rate) is applied to the top surface of the electrode film, while all other faces are flux free.

\subsection{The plastic electrode materials with different hardening behavior}

Here we examine how strain hardening in the electrode film affects the stress-capacity curves. We show in Fig. 2a the equivalent stress $\sigma_{e}$ and equivalent strain $\varepsilon_{e}$ curves of the electrode material during charge and discharge, where $\sigma_{e}=$ $\sqrt{\frac{3}{2}\left(\sigma_{i j}-\frac{1}{3} \sigma_{i j} \delta_{i j}\right)\left(\sigma_{i j}-\frac{1}{3} \sigma_{i j} \delta_{i j}\right)}$ and $\varepsilon_{e}=\sqrt{\frac{2}{3}\left(\varepsilon_{i j}-\frac{1}{3} \varepsilon_{i j} \delta_{i j}\right)\left(\varepsilon_{i j}-\frac{1}{3} \varepsilon_{i j} \delta_{i j}\right)}, \delta_{i j}=1$ if $i=j$ and $\delta_{i j}=0$ otherwise. The electrode material exhibits bilinear isotropic hardening during charge, and discharge induced unloading is elastic at the beginning, followed by plastic discharge. In Fig. $2 \mathrm{~b}$, we show the substrate's curvature $\kappa$ as a function of $\psi$ from FE calculation. If both the elastic modulus and the initial yield stress of the electrode material are the same (see Fig. 2a), the slopes of the resulted $\kappa-\psi$ response are the same as well in the elastic stage. In contrast, the plastic response in the $\kappa-\psi$ curves and the hysteresis loop area are affected by the hardening modulus $E_{t}$. We also observe from Fig. $2 \mathrm{~b}$ cyclic hardening in different charge/discharge cycles. The hysteresis loop reflects the influence of electrode materials' plastic dissipation on $\kappa$. In Fig. $2 \mathrm{c}$, we give the correlation between the curvature 'hardening' ( $\kappa_{t}$, the slope of $\kappa-\psi$ curve in the hardening region) and the electrode materials' hardening. We notice that the relationship between $K_{t} / K_{e}$ and $E_{t} / E_{0}$ does not follow a linear relationship. Large plastic deformation in the film affects the slope of the $\kappa / \kappa_{0}-\psi$ curves: greater $E_{t}$ leads to less plasticity in the electrode material. By comparing the film's biaxial stress from the FEM results and that from the Stoney equation, as shown in Fig. $2 \mathrm{~d}$, we see that the Stoney equation overestimates the stress in the film due to the large deformation, especially during large plastic deformation. From Fig. 2e, we also see that the thickness of the electrode film $h_{f}$ varies significantly as a function of concentration. Furthermore, it accumulates with cycling when we record $h_{f}$ at the same concentration $\psi$. The large volume change and resulted film thickness variation account for the deviation of real-time stress in electrode materials from Stoney equation based prediction.

\subsection{The elastic-plastic electrode with different initial yield stress}

Now we assume that the initial yield stress is proportional to the elastic modulus, that is, $\sigma_{y 0}=5 \% E_{0}$. In Fig. $3 a$, we extract the stress-strain curve of the film electrode material from the FE model. By changing the initial elastic modulus, we examine the effect of variation in yield stress on the curvature- $\psi$ curve. As shown in Fig. $3 \mathrm{~b}$, the slope of the $\kappa-\psi$ curves could be used as a measurement for the elastic modulus of the electrode material (Sethuraman et al., 2010). In Fig. 3c, we also show the averaged Cauchy stress in the electrode at different concentration $\psi$. During different charge/discharge cycles, there is also cyclic hardening behavior, which is a resultant of the implemented hardening behavior of the electrode material. As shown in Fig. 3d, the curvature at the turning point depends linearly on the initial yield strength. This feature may be utilized to determine the yield strength of electrode materials if one obtains the curvature-capacity curve.

\subsection{Predictability against experimental results}

As previously discussed, the Stoney equation is not suitable for silicon electrode material which exhibits large plastic deformation. A simple and effective method for getting electrode material properties is to realize a fully coupled electrochemical-mechanics FEM theory, and to adjust electrode material parameters by fitting experimental data. According to the atomistic simulation and experiment results shown in Fig. 1c and d, the properties of electrode materials are sensitive to lithium concentration. The plastic response changes with the state of charge $\psi$ (Fan et al., 2013; Ding et al., 2015; Wang et al., 2015). We take this effect into account and consider the influence of lithium concentration on electrode properties by adopting the Voce model (Voce, 1955). Other models could be conveniently implemented in the current computational scheme. In the Voce model, the yield stress $\sigma_{y}$ of the electrode material is now a function of state of charge, 


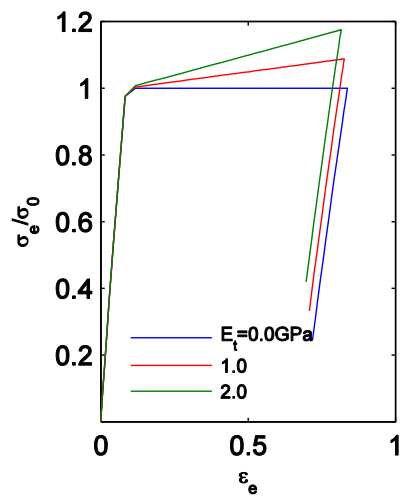

(a)

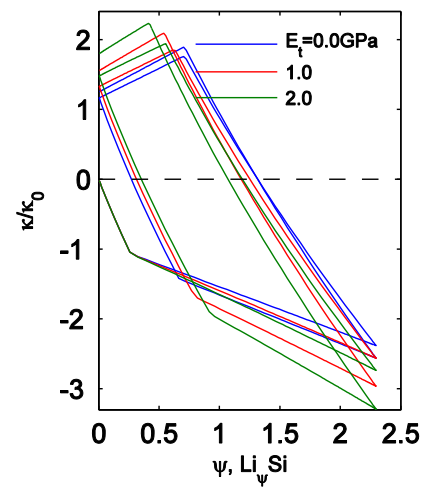

(b)

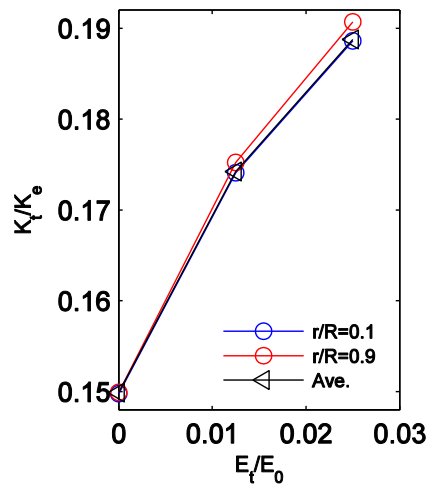

(c)

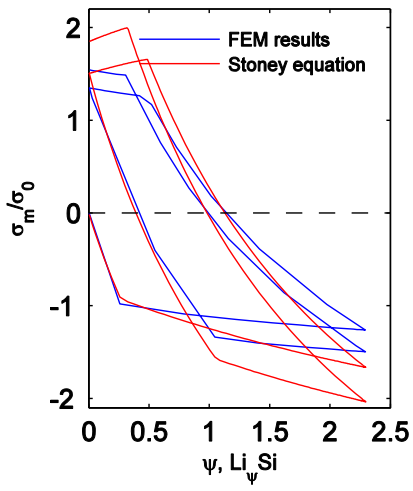

(d)

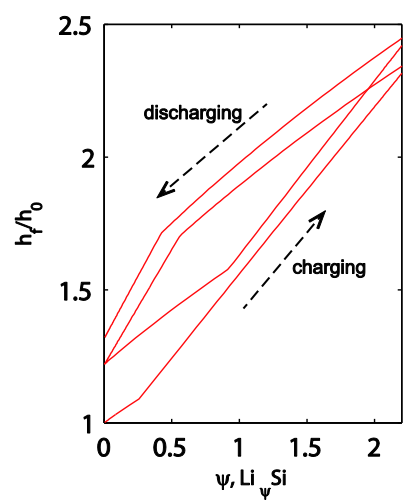

(e)

Fig. 2. The calculated substrate's curvature $\kappa$ and film's biaxial stress evolution $\sigma_{m}$ in two charge/discharge cycles using the finite deformation algorithms with different hardening modulus $E_{t}$. Here the electrode film is assumed to follow a bilinear isotropic hardening. The Cauchy stress is normalized by the initial yield stress $\sigma_{0}=\sigma_{y}=5 \mathrm{GPa}$. The curvature is normalized by $\kappa_{0}=\frac{6\left(1-v_{s}\right) h_{f}}{E_{s} h_{s}^{2}} \sigma_{0}$ according to the Stoney equation (Eq. (22)). (a) Different constitutive behavior of the electrode extracted from one material point in the FE model. (b) The average curvature of the substrate vs. $\psi$ along the radius from FE calculations. (c) The slope of the $\kappa / \kappa_{0}-\psi$ curves versus the normalized hardening modulus, where $K=\frac{d\left(\kappa / K_{0}\right)}{d \psi}$, and $K_{e}$ and $K_{t}$ are the slopes of the $\kappa / \kappa_{0}-\psi$ curve during the elastic and the plastic stage in Fig. $2 \mathrm{~b}$, respectively. (d) The averaged biaxial stress vs. $\psi$ in the film from FE calculations and that from the Stoney equation when $E_{t}=2.0 \mathrm{GPa}$. (e) The evolution of electrode film's thickness $h_{f}$ as a function of $\psi$, in which $h_{0}$ is the initial thickness of the film.

$$
\frac{\sigma_{y}}{\sigma_{y 0}}=1+\frac{\psi(t)}{\psi_{\max }} R_{0}+R_{\infty}\left[1-e^{-\frac{\psi(t)}{\psi_{\max }} b}\right] .
$$

We show in Fig. 4a the described yield stress $\sigma_{y}$ of the electrode material as a function of $\psi$ by the Voce model in Eq. (24). The parameters used in the model are given as follows, $\sigma_{y 0}=2.0 \mathrm{GPa}, R_{0}=0, R_{\infty}=-0.75, b=5$. In Fig. $4 \mathrm{~b}$, we compare the experimental results from Sethuraman et al. (2010) with our FEM calculations using the same group of parameters for Fig. 4a. For the simple situation that $R_{\infty}=0$, the yield stress depends linearly on lithium fraction $\psi(t)$. When $R_{0}<0$, there is a lithiation softening effect during charging. Indeed, observations on amorphous silicon electrode materials do see perceivable stress-softening behavior (Sethuraman et al., 2010; Soni et al., 2012). For lithiation strengthening materials (Sethuraman et al., 2012; Soni et al., 2012; Ding et al., 2015), we may let $R_{0}>0$ to capture the effect. Corresponding results are shown in Fig. 4c and d.

\section{Interface delamination in the film-substrate structure}

In the previous section, we assume that the film electrode is perfectly adhered to the substrate. For high charge ratio electrode materials, the stress on the order of GPa is a serious challenge to the interface (Shi et al., 2016; Yang et al., 2018). Once there is delamination or interfacial sliding between the film and the substrate, the Stoney equation is also not applicable. In this section, we discuss the influence of the interface's plastic dissipation and failure on in situ stress measurements. 


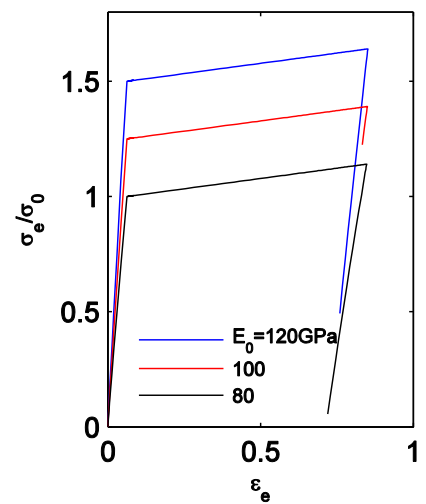

(a)

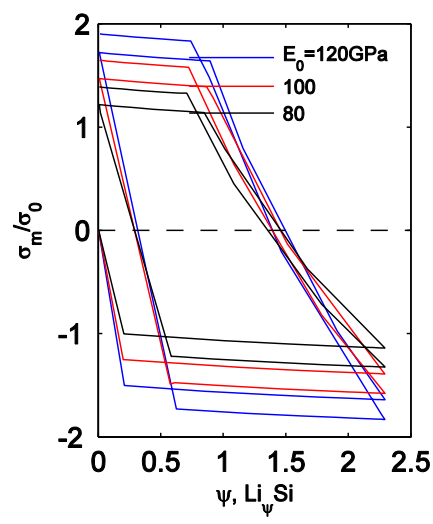

(c)

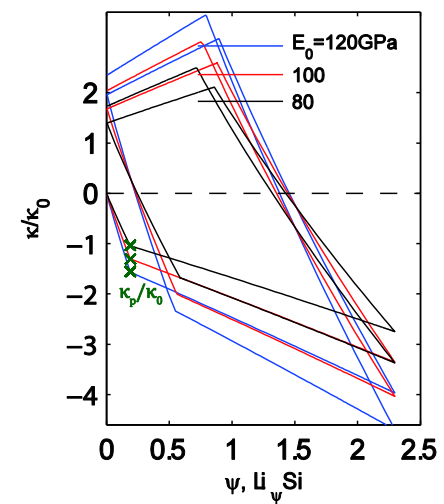

(b)

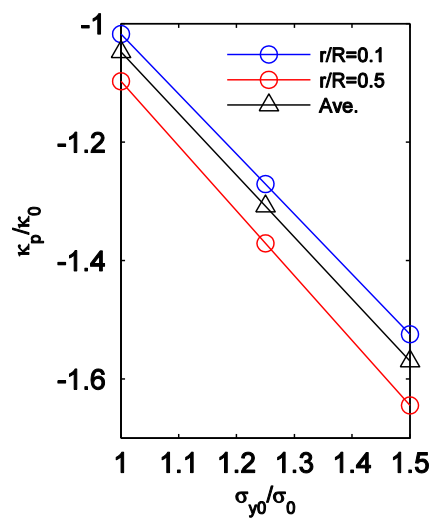

(d)

Fig. 3. Curvature $\kappa$ and film's biaxial stress $\sigma_{m}$ in two charge/discharge cycles. Here the electrode film has different yield stress $\sigma_{y 0} / E_{0}=5 \%$ and hardens linearly with $E_{t}=1.0 \mathrm{GPa}$. The Cauchy stress and the curvature are normalized by the initial yield stress $\sigma_{0}=5 \mathrm{GPa}$ and $\kappa_{0}=\frac{6\left(1-v_{s}\right) h_{f}}{E_{s} h_{s}^{2}} \sigma_{0}$, respectively. (a) Constitutive behavior of three electrode materials (different $E_{0}$ ) extracted from a material point in the FE model. (b) The average curvature of the substrate vs. $\psi$ along the radial direction from FE calculations. Here $\kappa_{p}$ is the curvature at the transitional point due to initial yielding. (c) The averaged biaxial stress vs. $\psi$ in the film from FE calculations. (d) The relationship between $\kappa_{p} / \kappa_{0}$ and $\sigma_{y_{0}} / \sigma_{0}$.

As seen in Fig. 1a, a cohesive layer bonds the film electrode to the substrate for in situ stress measurement. Ti and $\mathrm{Cu}$ are usually used as bonder materials because of their high cohesive properties and large plastic dissipation (Sethuraman et al., 2010; Soni et al., 2012). To investigate the influence of deformation in the cohesive interface on in situ stress measurements, we adopt the 4-node axisymmetric cohesive element in ABAQUS (COHAX4) to simulate interface delamination (ABAQUS/Standard, 2013). Different types of interfacial constitutive model (Needleman, 1990; Xu and Needleman, 1994; Camacho and Ortiz, 1996; Wei and Anand, 2004; Wei, 2014) may be employed. Here we consider the one with normal stress and shear stress response demonstrated in Fig. 5a: the initial response of the cohesive interface is linear, with an elastic stiffness $K_{n}$ in the normal direction and $K_{s}$ in the tangential shear direction. Damage starts when either the normal or the shear stress reaches their respective threshold, $t_{n}^{\max }$ and $t_{s}^{\max }$. During the stage of damage evolution, a scalar variable $D$ represents the overall damage in the interface material. The interface degrades linearly with damage $D$ as it grows from 0 (intact initial state) to 1 (complete failure). Upon unloading, interfacial stress returns to the origin, as illustrated by the dashed line, and the elastic stiffness is multiplied by the factor $(1-D)$. We consider the case that the initial normal failure strength is significantly greater than the shear strength, with $t_{n}^{\max }=1 \mathrm{GPa}$ and $K_{n}=100 \mathrm{GPa}$. The tangential properties will be given in the particular applications to be presented. The bonder's separation $\delta$ is normalized by its initial thickness $h_{b 0}$, which is assumed to be $h_{b 0}=1 \mu \mathrm{m}$.

\subsection{The influence of interface stiffness}

We first explore the influence of interface shear stiffness on the curvature-capacity response of a thin film battery. As seen in Fig. 5b and c, the interfacial stiffness $K_{s} / E_{0}$ has a small impact on the slope in the $\kappa-\psi$ response when $K_{s} / E_{0}>0.1$. Only if $K_{S} / E_{0}<0.01$ and also nearby the edge of the film $(r / R=0.9)$, the effect of interface stiffness becomes perceivable. In Fig. $5 \mathrm{~d}$, we show the slope $K_{e}$ of the $\kappa-\psi$ curves at the elastic stage as a function of $K_{s} / E_{0}$. For better demonstration, $K_{e}$ is 


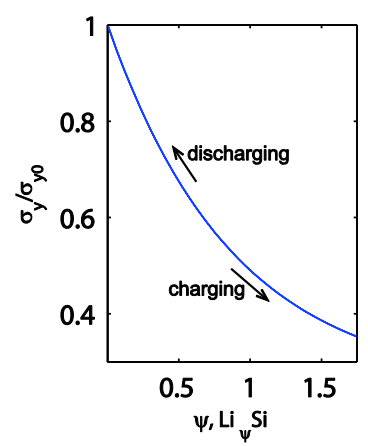

(a)

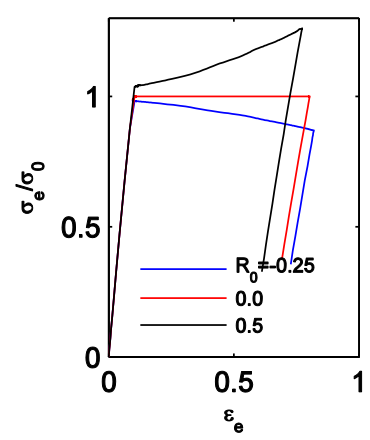

(c)

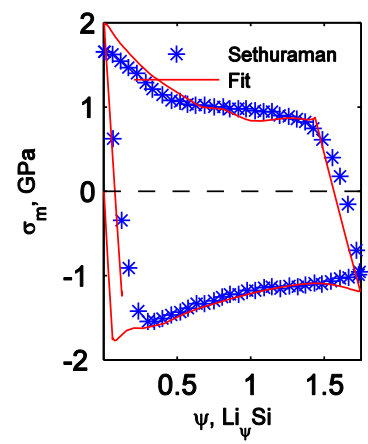

(b)

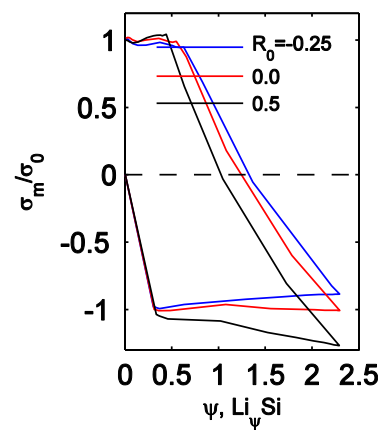

(d)

Fig. 4. Dependence of yield strength with lithium fraction. (a) The yield stress $\sigma_{y}$ of the electrode material as a function of $\psi$ in the Voce model (see Eq. (24)) during charging and discharging. (b) The numerical results against experimental data from Sethuraman et al. (2010). (c) Mechanical behavior with different yield stress dependence from FE model. (d) The averaged biaxial stress vs. $\psi$ in the film from FE calculations.

normalized by the corresponding slope $K_{0}$ of the $\kappa-\psi$ curve from the perfectly bonded film-substrate system. With small interface stiffness and also when $r \rightarrow R$, we see $K_{e} / K_{0}$ is apparently affected by the interfacial elastic deformation, which is consistent with previous analysis on how interfacial elasticity changes a multilayer plate's resistance to bending (Peng and Wei, 2016).

\subsection{The influence of interfacial strength}

Now we discuss the influence of interfacial shear strength on the curvature-capacity relationship. Typically, the bonder materials have low strength. For example, $\mathrm{Cu}$ bonder has a shear strength lower than $100 \mathrm{MPa}$, and a fracture toughness lower than $2 \mathrm{~J} / \mathrm{m}^{2}$. Meanwhile, both the strength and fracture toughness drop when lithium concentration increases during the charge process (Stournara et al., 2013). In order to discuss the competition between electrode materials' plastic response and interfacial plasticity, we consider the interface of different shear strength. Its effect to the curvature of the electrode during charging-discharging cycles is shown in Fig. 6a. We see the smallest curvature (negative value) increases with the interfacial shear strength. In Fig. 6b, we show the curvature vs. concentration relationship at different positions along the radial direction. The biaxial Cauchy stress $\sigma_{m}$ as a function of interfacial shear strength is shown in Fig. 6c. Here the calculation is done at the point with the smallest $\kappa / \kappa_{0}$ (negative value) in Fig. $6 \mathrm{~b}$. The maximum stress increases with the shear strength in the center $(r / R=0.1)$ where its deformation is still elastic. The same trend is also seen at the edge $(r / R=0.9)$ where plastic deformation dominates. However, the behavior is totally different in the transitional region, i.e. $r / R \sim 0.5$, where elastic-plastic deformation coexists.

\subsection{Interfacial delamination with plastic dissipation}

When shearing of the interface is significant, the interface starts to fail. In Fig. 7a, we show the curvature in the region $(r / R=0.8)$ when different failure separation $\frac{\delta_{f}}{h_{b 0}}$ is used. When $\frac{\delta_{f}}{h_{b 0}}$ is large, the average curvature is the same as that in Fig. 6a. For $\delta_{f}$ smaller than $h_{b 0}$, curvature $\kappa$ reduces, which is caused by the softening in the interface. For the convenience of better view, we show in Fig. $7 \mathrm{~b}$ and $\mathrm{c}$ the amplified edge of the film-substrate structure. Fig. $7 \mathrm{~b}$ gives the simulated deformation in the electrode film by using $\frac{\delta_{f}}{h_{b 0}}=0.5$. We see clearly interfacial delamination due to excessive sliding during 


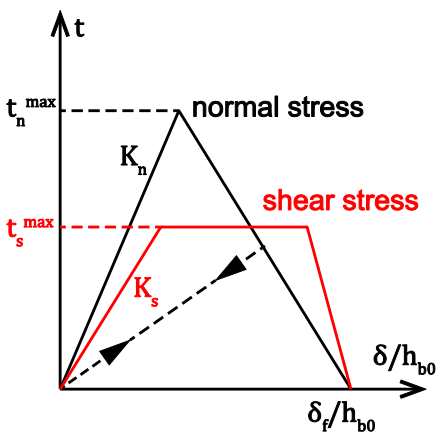

(a)

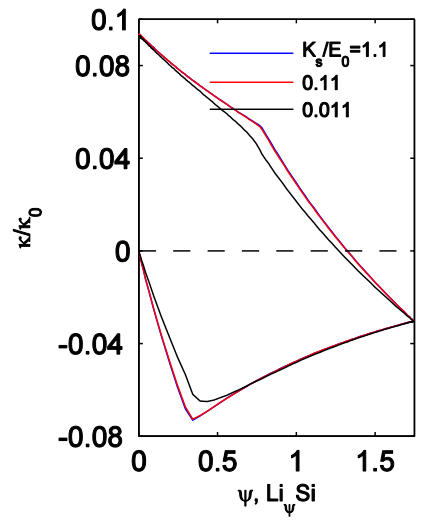

(c)

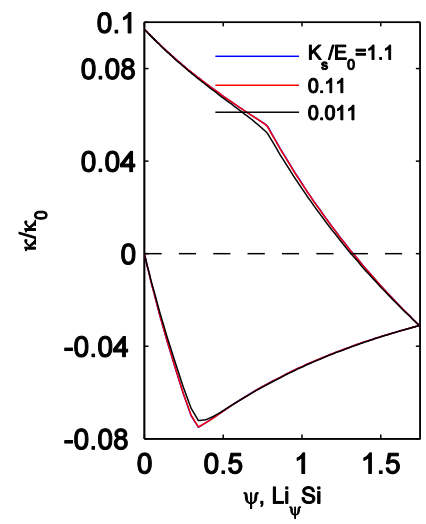

(b)

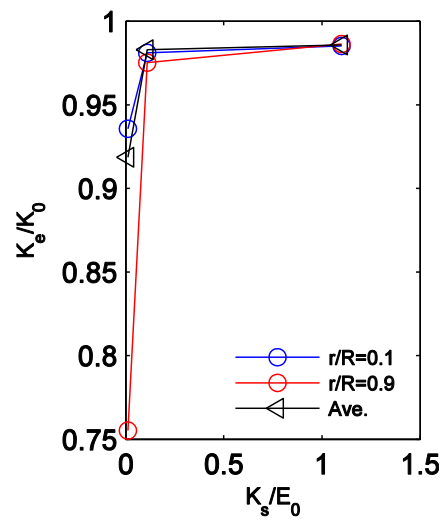

(d)

Fig. 5. Curvature evolution with different interfacial stiffness. Here $E_{0}$ and $K_{s}$ are the modulus and the stiffness of the electrode material and the interface, respectively. (a) The constitutive relationship of the cohesive element. (b) \& (c) The substrate's curvature vs. $\psi$ from FE calculations at the position $r / R=0.1$ and 0.9 , respectively. The difference is negligible for $K_{s} / E_{0}>0.1$. (d) The slope of $\kappa-\psi$ curves in the initial linear stage at different position $r / R$ along the radial direction. Here $K_{e}$ is the slope of the $\kappa-\psi$ curves in the initial linear stage, and $K_{0}$ is the slope of $\kappa-\psi$ curve when the film is perfectly tied to the substrate.

discharge. In Fig. 7c, we show the shear stress evolution in the film electrode during charging and discharging. For better presentation, shear stress in the substrate is not shown. During charging, we see propagation of the maximum shear stress, from $(r / R \sim 0.8)$ to the center of the film $(r / R \sim 0)$. From ii to iv in Fig $7 c$, we see shear stress relaxation caused by interfacial failure. The opposite shear stress continues to grow and causes continuing crack propagation during discharging. As the failed front moves, the film could be lifted off from the substrate. At the end of the discharging process, a large portion of the interface is delaminated, as shown in Fig. $7 \mathrm{c}$ (viii).

\section{Conclusions}

In this paper, we develop a FE procedure for large plastic deformation to solve the strongly electrochemical-mechanical coupled problem for the high capacity batteries, which can be used to help understand the stress evolution in electrode materials. It may also be used to extract the properties of electrode materials if corresponding experimental stress-capacity data are available for calibration. We apply the model and the numerical method to demonstrate the stress-capacity change in response to different hardening modulus and initial yield stress of the electrode material. By taking the concentrationdependent elastic-plastic behavior of electrode materials (Fig. 1d and e) into account, we are able to fit the stress-capacity curve from experiments. We further show that interfacial delamination has a significant effect on the accuracy of in situ stress measurements. The numerical procedure should be able to, in combination with experimental measurements, reproduce the electrochemical-mechanical process in realistic battery systems. It may also be used to extract material parameters of the electrode material and the interface properties in a film-substrate battery system. 


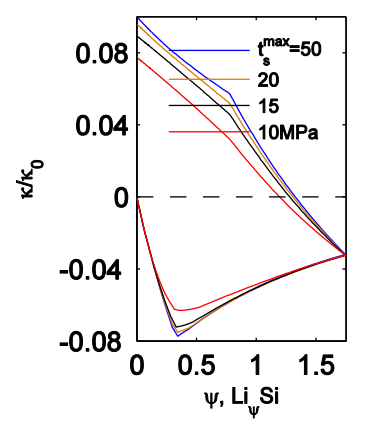

(a)

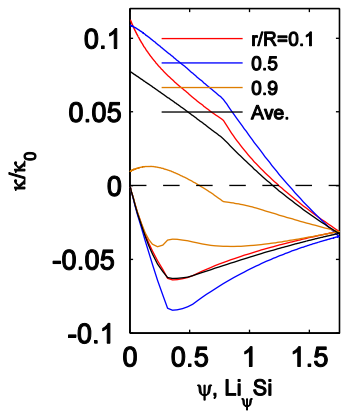

(b)

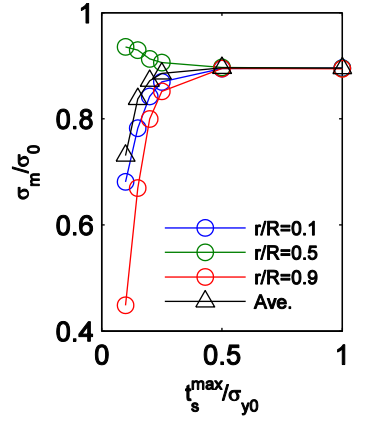

(c)

Fig. 6. Curvature evolution with different initial interfacial shear strength $t_{s}{ }^{\max }$. (a) The average curvature vs. $\psi$ from FE model. (b) The curvature in different radial positions and the averaged value along the radius during a charge/discharge cycle. (c) The calculated biaxial Cauchy stress $\sigma_{m}$ in the charging stage using Eq. (22) and the smallest $\kappa / \kappa_{0}$ in (b).

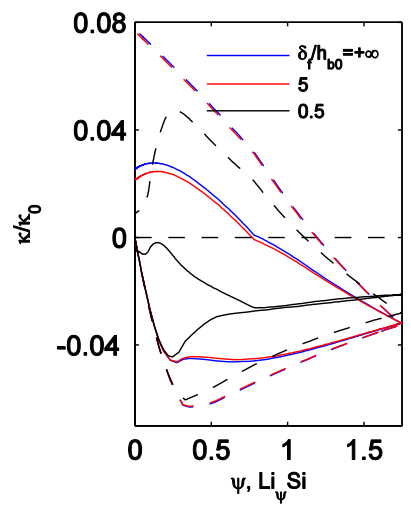

(a)

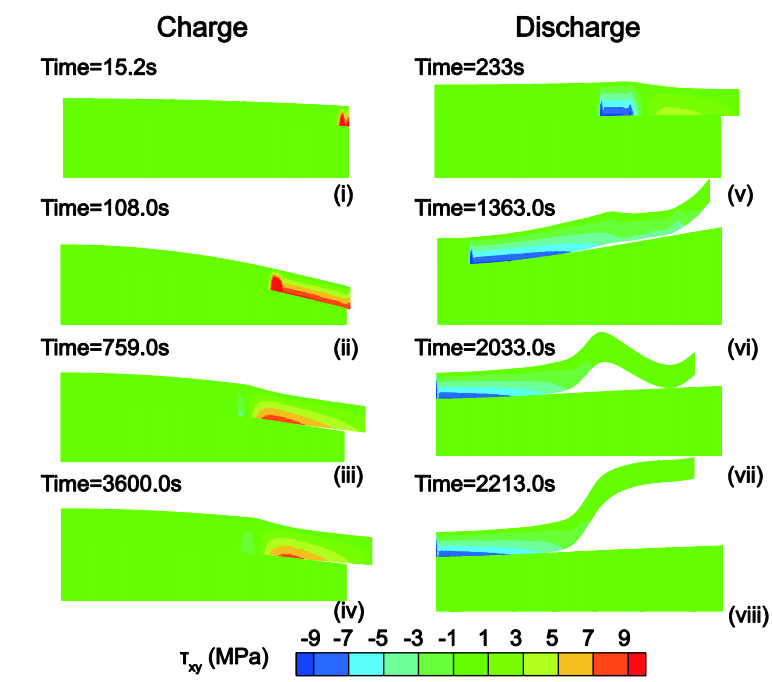

(c)

Discharge

$m e=233 \mathrm{~s}$

(vi)

(vii)

(viii)

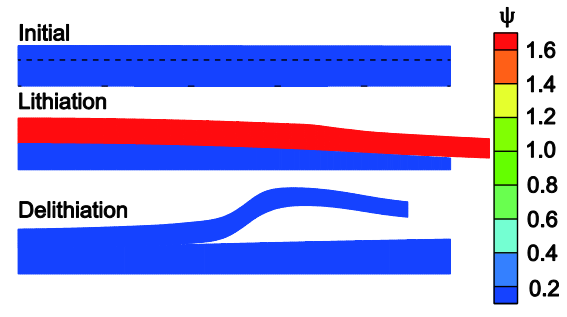

(b)

7. The influence of the interfacial fracture energy on the substrate's curvature evolution. Here the initial interfacial shear strength is $t^{\max }=10 \mathrm{MPa}$ (a) The substrate's curvature vs. $\psi$ from FE model at the position of $r / R=0.8$ (the solid line) and its average value along the radial direction (the dotted line). (b) FE model's deformation map. Here the structure in the thickness direction has been amplified by a factor of 100 . (c) The shear stress map in the film in different charging and discharging stage. The dimension in the thickness direction is 30 times larger than that in the radial direction. 


\section{Acknowledgments}

The authors acknowledge support from the National Natural Science Foundation of China (NSFC) (Grant no. 11425211), the Strategic Priority Research Program of the Chinese Academy of Sciences (XDB22020200). YTC would like to thank US National Science Foundation award 1355438 "Powering the Kentucky Bioeconomy for a Sustainable Future."

\section{Supplementary materials}

Supplementary material associated with this article can be found, in the online version, at doi:10.1016/j.jmps.2018.04.006.

\section{References}

ABAQUS/Standard, 2013. SIMULIA, Providence, RI.

Anand, L., 2012. A Cahn-Hilliard-type theory for species diffusion coupled with large elastic-plastic deformations. J. Mech. Phys. Solids 60 (12), $1983-2002$.

Bhandakkar, T.K., Chason, E., Gao, H., 2010. Analytical model of transient compressive stress evolution during growth of high diffusivity thin films on substrates. Philos. Mag. 90 (22), 3037-3048.

Boukamp, B.A. Lesh, G.C. Huggins, R.A., 1981. All-solid lithium electrodes with mixed-conductor matrix. J. Electrochem. Soc, 128 (4), 725-729.

Bourderau, S., Brousse, T., Schleich, D.M., 1999. Amorphous silicon as a possible anode material for Li-ion batteries. J. Power Sources 81, $233-236$.

Bower, A.F., Guduru, P.R., Sethuraman, V.A., 2011. A finite strain model of stress, diffusion, plastic flow, and electrochemical reactions in a lithium-ion half-cell. J. Mech. Phys. Solids 59 (4), 804-828.

Brantley, W.A., 1973. Calculated elastic constants for stress problems associated with semiconductor devices. J. Appl. Phys. 44 (1), $534-535$.

Brassart, L., Zhao, K., Suo, Z., 2013. Cyclic plasticity and shakedown in high-capacity electrodes of lithium-ion batteries. Int. J. Solids Struct. 50 (7), 1120-1129.

Camacho, G.T., Ortiz, M., 1996. Computational modeling of impact damage in brittle materials. Int. J. Solids Struct. 33, 2899-2938.

Carslaw, H.S., Jaeger, J.C., 1959. In: Conduction of Heat in Solids, vol. 2. Clarendon press, Oxford, pp. 8-13.

Chan, C.K., Peng, H., Liu, G., Mcllwrath, K., Zhang, X.F., Huggins, R.A., Cui, Y., 2008. High-performance lithium battery anodes using silicon nanowires. Nat. Nanotechnol. 3 (1), 31-35.

Cheng, Y.T., Verbrugge, M.W., 2009. Evolution of stress within a spherical insertion electrode particle under potentiostatic and galvanostatic operation. J. Power Sources 190, 453-460.

Chester, S.A., Di Leo, C.V., Anand, L., 2015. A finite element implementation of a coupled diffusion-deformation theory for elastomeric gels. Int. J. Solids Struct. 52, 1-18.

Crisfield, M.A., 1997. Non-linear Finite Element Analysis of Solids and Structures Vol. 2: Advanced Topic. John Wiley\&Sons, Chichester.

Ding, B., Li, X., Zhang, X., Wu, H., Xu, Z., Gao, H., 2015. Brittle versus ductile fracture mechanism transition in amorphous lithiated silicon: from intrinsic nanoscale cavitation to shear banding. Nano Energy 18, 89-96.

Di Leo, C.V., Rejovitzky, E., Anand, L., 2014. A Cahn-Hilliard-type phase-field theory for species diffusion coupled with large elastic deformations: application to phase-separating Li-ion electrode materials. J. Mech. Phys. Solids 70, 1-29.

Duan, C., Wei, Y., 2017. Theory on bending in cantilever beams with adsorbed islands. J. Appl. Mech. 84 (7), 071006.

Fan, F., Huang, S., Raju, M., Datta, D., Shenoy, V.B., Van Duin, A.C., Zhang, S., Zhu, T., 2013. Mechanical properties of amorphous LixSi alloys: a reactive force field study. Model. Simul. Mater. Sci. Eng. 21074002.

Haftbaradaran, H., Xiao, X., Verbrugge, M.W., Gao, H., 2012. Method to deduce the critical size for interfacial delamination of patterned electrode structures and application to lithiation of thin-film silicon islands. J. Power Sources 206, 357-366.

He, Y., Yu, X., Li, G., Wang, R., Li, H., Wang, Y., Gau, H., Huang, X., 2012. Shape evolution of patterned amorphous and polycrystalline silicon microarray thin film electrodes caused by lithium insertion and extraction. J. Power Sources 216, 131-138.

Huggins, R.A., 1999. Lithium alloy negative electrodes. J. Power Sources 81, 13-19.

Hughes, T.J.R., Simo, J.C., 1998. Computational Inelasticity. Springer-Verlag, New York.

Hughes, T.J., 1984. Numerical implementation of constitutive models: rate-independent deviatoric plasticity. In: Theoretical Foundation for Large-Scale Computations for Nonlinear Material Behavior, pp. 29-63.

Jia, Z., Li, T., 2016. Intrinsic stress mitigation via elastic softening during two-step electrochemical lithiation of amorphous silicon. J. Mech. Phys. Solids 91, 278-290.

Klinsmann, M., Rosato, D., Kamlah, M., McMeeking, R.M., 2016. Modeling crack growth during Li extraction in storage particles using a fracture phase field approach. J. Electrochem. Soc. 163 (2), A102-A118.

Liang, B., Liu, Y., Xu, Y., 2014. Silicon-based materials as high capacity anodes for next generation lithium ion batteries. J. Power Sources 267, 469-490.

Levitas, V.I., Attariani, H., 2014. Anisotropic compositional expansion in elastoplastic materials and corresponding chemical potential: Large-strain formulation and application to amorphous lithiated silicon. J. Mech. Phys. Solids 69, 84-111.

Li, J.C.M., 1978. Physical chemistry of some microstructural phenomena. Metall. Trans. A 9 (10), 1353-1380.

Maranchi, J.P., Hepp, A.F., Evans, A.G., Nuhfer, N.T., Kumta, P.N., 2006. Interfacial properties of the a-Si/Cu: active-inactive thin-film anode system for lithium-ion batteries. J. Electrochem. Soc. 153 (6), A1246-A1253.

McMeeking, R.M., Rice, J.R., 1975. Finite-element formulations for problems of large elastic-plastic deformation. Int. J. Solids Struct. 11 (5), 601-616.

Needleman, A., 1990. An analysis of decohesion along an imperfect interface. Non-Linear Fract. 42, 21-40.

Pan, J., Zhang, Q., Li, J., Beck, M.J., Xiao, X., Cheng, Y.T., 2015. Effects of stress on lithium transport in amorphous silicon electrodes for lithium-ion batteries. Nano Energy 13, 192-199.

Peng, S., Wei, Y., 2016. On the influence of interfacial properties to the bending rigidity of layered structures. J. Mech. Phys. Solids 92, 278-296.

Pharr, M., Choi, Y.S., Lee, D., Oh, K.H., Vlassak, J.J., 2016. Measurements of stress and fracture in germanium electrodes of lithium-ion batteries during electrochemical lithiation and delithiation. J. Power Sources 304, 164-169.

Pharr, M., Suo, Z., Vlassak, J.J., 2013. Measurements of the fracture energy of lithiated silicon electrodes of Li-ion batteries. Nano Lett. 13 (11), $5570-5577$.

Sethuraman, V.A., Chon, M.J., Shimshak, M., Srinivasan, V., Guduru, P.R., 2010. In situ measurements of stress evolution in silicon thin films during electrochemical lithiation and delithiation. J. Power Sources 195 (15), 5062-5066.

Sethuraman, V.A., Chon, M.J., Shimshak, M., Van Winkle, N., Guduru, P.R., 2010. In situ measurement of biaxial modulus of Si anode for Li-ion batteries. Electrochem. Commun. 12 (11), 1614-1617.

Sethuraman, V.A., Van Winkle, N., Abraham, D.P., Bower, A.F., Guduru, P.R., 2012. Real-time stress measurements in lithium-ion battery negative -electrodes. J. Power Sources 206, 334-342.

Shenoy, V.B., Johari, P., Qi, Y., 2010. Elastic softening of amorphous and crystalline Li-Si phases with increasing Li concentration: a first-principles study. J. Power Sources 195 (19), 6825-6830.

Shi, F., Song, Z., Ross, P.N., Somorjai, G.A., Ritchie, R.O., Komvopoulos, K., 2016. Failure mechanisms of single-crystal silicon electrodes in lithium-ion batteries. Nat. Commun. 7, 11886.

Soni, S.K., Sheldon, B.W., Xiao, X., Verbrugge, M.W., Dongjoon, A., Haftbaradaran, H., Huajian, G., 2011. Stress mitigation during the lithiation of patterned amorphous Si islands. J. Electrochem. Soc. 159 (1), A38-A43. 
Soni, S.K., Sheldon, B.W., Xiao, X., Bower, A.F., Verbrugge, M.W., 2012. Diffusion mediated lithiation stresses in Si thin film electrodes. J. Electrochem. Soc. 159 (9), A1520-A1527.

Stoney, G.G., 1909. The tension of metallic films deposited by electrolysis. In: Proceedings of the Royal Society of London, Series A, 82, pp. 172-175.

Stournara, M.E., Xiao, X., Qi, Y., Johari, P., Lu, P., Sheldon, B.W., Gao, H., Shenoy, V.B., 2013. Li segregation induces structure and strength changes at the amorphous Si/Cu interface. Nano Lett. 13 (10), 4759-4768.

Szczech, J.R., Jin, S., 2011. Nanostructured silicon for high capacity lithium battery anodes. Energy Environ. Sci. 4 (1), $56-72$.

Voce, E., 1955. A practical strain-hardening function. Metallurgia 51 (307), 219-226.

Wang, X., Fan, F.F., Wang, J.J., Wang, H.R., Tao, S.Y., Yang, A., Liu, Y., Chew, H.B., Mao, S.X., Zhu, T., Xia, S.M., 2015. High damage tolerance of electrochemically lithiated silicon. Nature Commun. 6, 8417

Wei, Y.J., Anand, L., 2004. Grain-boundary sliding and separation in polycrystalline metals: application to nanocrystalline fcc metals. J. Mech. Phys. Solids 52 (11), 2587-2616.

Wei, Y.J., 2014. A stochastic description on the traction-separation law of an interface with non-covalent bonding. J. Mech. Phys. Solids 70, 227-241.

Wei, Y.J., Bower, A.F., Gao, H., 2010. Analytical model and molecular dynamics simulations of the size dependence of flow stress in amorphous intermetallic nanowires at temperatures near the glass transition. Phys. Rev. B 81, 125402.

Wen, J., Wei, Y., Cheng, Y.T., 2018. Examining the validity of Stoney-equation for in-situ stress measurements in thin film electrodes using a large-deformation finite-element procedure. J. Power Sources 387, 126-134.

Winter, M., Besenhard, J.O., 1999. Electrochemical lithiation of tin and tin-based intermetallics and composites. Electrochim. Acta 45 (1), $31-50$.

Xu, X.P., Needleman, A., 1994. Numerical simulations of fast crack growth in brittle solids. J. Mech. Phys. Solids 42 (9), $1397-1434$.

Yang, F., 2005. Interaction between diffusion and chemical stresses. Mater. Sci. Eng. A 409 (1), 153-159.

Yang, H., Fan, F., Liang, W., Guo, X., Zhu, T., Zhang, S., 2014. A chemo-mechanical model of lithiation in silicon. J. Mech. Phys. Solids 70, 349-361.

Yang, L., Chen, H.S., Jiang, H., Wei, Y.J., Song, W.L., Fang, D.L., 2018. Failure mechanism of 2D silicon film anodes: in-situ observations and simulations on crack evolution. Chem. Commun. doi:10.1039/c7cc09708E.

Zhang, X., Krischok, A., Linder, C., 2016. A variational framework to model diffusion induced large plastic deformation and phase field fracture during initial two-phase lithiation of silicon electrodes. Comput. Meth. Appl. Mech. Eng. 312, 51-77.

Zhang, X., Sastry, A.M., Shyy, W., 2008. Intercalation-induced stress and heat generation within single lithium-ion battery cathode particles. J. Electrochem. Soc. 155 (7), A542-A552.

Zhang, X., Shyy, W., Sastry, A.M., 2007. Numerical simulation of intercalation-induced stress in Li-ion battery electrode particles. J. Electrochem. Soc. 154 (10), A910-A916.

Zhang, X.Y., Song, W.L., Liu, Z., Chen, H.S., Li, T., Wei, Y., Fang, D.N., 2017. Geometric design of micron-sized crystalline silicon anodes through in situ observation of deformation and fracture behaviors. J. Mater. Chem. A 5 (25), 12793-12802.

Zhao, K., Pharr, M., Vlassak, J.J., Suo, Z., 2011. Inelastic hosts as electrodes for high-capacity lithium-ion batteries. J. Appl. Phys. 109 (1), 31.

Zhao, K., Tritsaris, G.A., Pharr, M., Wang, W.L., Okeke, O., Suo, Z., Vlassak, J.J., Kaxiras, E., 2012. Reactive flow in silicon electrodes assisted by the insertion of lithium. Nano Lett. 12 (8), 4397-4403. 